\title{
Longitudinal Myelitis as a Rare First Symptom of Lupus in Overlap Syndrome
}

\author{
Beata WILCZEK-PARADOWSKA, ${ }^{1}$ Michal PARADOWSKI ${ }^{2}$ \\ ${ }^{1}$ Department of Neurology, $4^{\text {th }}$ Military Hospital With Polyclinic, Wroclaw, Poland \\ ${ }^{2}$ Department of Neurology, Medical University Wroclaw, Wroclaw, Poland
}

\begin{abstract}
In this article, we present a 46-year-old female patient who had been diagnosed with Sjögren's syndrome for 14 years. After initial non-specific signs of abdominal and back pain, neurological symptoms were observed, leading to a final diagnosis of overlap syndrome of neuropsychiatric systemic lupus erythematosus and Sjögren's syndrome. Inflammatory myelopathy, referred to as longitudinal myelitis, caused severe and persistent neurological deficit in our patient. The provided magnetic resonance imaging scans of the spine show initial and one-year follow-up changes. Thus, we report rare and severe initial symptoms of lupus.

Keywords: Systemic lupus erythematosus; magnetic resonance imaging; Sjögren's syndrome.
\end{abstract}

An overlap syndrome is a comorbidity of two connective tissue diseases, meeting their diagnostic criteria. Their simultaneous diagnosis is not necessary. ${ }^{1}$ In the course of connective tissue diseases, one of the possible symptoms is an affection of the nervous system; and in a case of systemic lupus erythematosus (SLE), it leads to derivation of neuropsychiatric SLE as a variant of SLE with separate diagnostic criteria. One of its symptoms is myelitis, ${ }^{2}$ usually affecting a single segment of the spine as transverse myelitis. When the inflammatory process spans over adjacent segments of the spine, it is referred to as longitudinal myelitis. ${ }^{3}$ Although most of the cases of longitudinal myelitis described in connective tissue diseases concern SLE, ${ }^{4}$ there are publications describing this condition in other diseases from this group, such as Sjögren's syndrome (SS). ${ }^{5}$ The combination of fast progressing myelitis symptoms and an extensive inflammatory process affecting most of the spine with severe neurologic deficit is sometimes specified as catastrophic longitudinal myelitis. ${ }^{6}$

In this article, we present a female patient with catastrophic longitudinal myelitis secondary to neuropsychiatric SLE/SS overlap syndrome as a picture of rare and severe clinical course of lupus.

\section{CASE REPORT}

A 46-year-old female patient, who had been diagnosed with SS for 14 years, with a history of arthritis and leucopenia, was admitted to a neurological ward due to a weakness of lower limbs lasting one day. The symptoms were preceded by abdominal pain, followed by lower back pain, generalized weakness, and chills. Until then, SS had been treated with methotrexate (20 mg daily) and prednisone (15 mg daily). Physically flaccid inferior paraparesis with areflexia, sensory deficit in all modalities below 
$\mathrm{T}_{11}$ dermatome, and malar rash were detected. Within two days after admission; confusion, meningeal irritation signs, inferior paraplegia with urine incontinence, and superior paraparesis were observed. In magnetic resonance imaging (MRI) scans of the spinal cord, there was hyperintense signal on $\mathrm{T}_{2}$-weighted images between the third cervical vertebra and conus medullaris. In brain MRI scans, bilateral, subcortical multiple foci of hyperintense signal visible in $T_{2}$, fluid attenuated inversion recovery, and diffusionweighted imaging sequences were described (Figure 1a, b, d, e). Elevated anti-SS-related antigen $\mathrm{A}$, anti-SS-related antigen $\mathrm{B}$, anti-double stranded deoxyribonucleic acid, anti-nucleosome, anti-histone antibody titers, decreased serum complement 3 and complement 4 levels, and polyclonal hypergammaglobulinemia were detected as well as elevated protein level in the cerebrospinal fluid. Cerebrospinal fluid bacterial and fungal cultures were all negative. C-reactive protein concentration was elevated and erythrocyte sedimentation rate was increased. High-dose intravenous methylprednisolone (1000 mg daily) and cyclophosphamide (100 mg daily) were administered and improvement in consciousness level, superior paraparesis, and meningeal irritation signs resolution were observed. In follow-up MRI scans of the spinal cord, the presence of hyperintense $\mathrm{T}_{2}$ signal was still described from $\mathrm{T}_{2}$ to conus medullaris (Figure $1 \mathrm{c}$ ). At discharge, the patient was treated with cyclophosphamide (100 mg daily) and prednisone (40 mg daily). Follow-up was scheduled every three months. Neurological examination after one year revealed neither inferior paraparesis nor bladder control improvement and only minor improvement in superficial sensation below $\mathrm{T}_{11}$ dermatome. The subsequent MRI scans of the spine showed atrophy of the thoracic and lumbar spine, while medullar cone seemed to be preserved (Figure 1f, g). Neurological assessment two years after the onset of longitudinal myelitis revealed no improvement in comparison to the previous assessment.

\section{DISCUSSION}

Coexistence of SS and SLE is not rare. According to Manoussakis et al., ${ }^{7}$ this overlap syndrome constitutes between 8 to $30 \%$ of the overall number of SLE cases. The particular sequence of disease development in this overlap syndrome was described by Szanto et al. ${ }^{8}$ and for patients with primary SS, it amounts to 15\%. Yang et al. ${ }^{9}$ examined risk factors of SLE development in patients with SS. They demonstrated that independent risk factors were low levels of complement components and young age at the onset of SS. According to Pan et al., ${ }^{10}$ the onset of SLE as a second disease in connective tissue disease overlap syndrome appears later in comparison to SLE as a single disease.
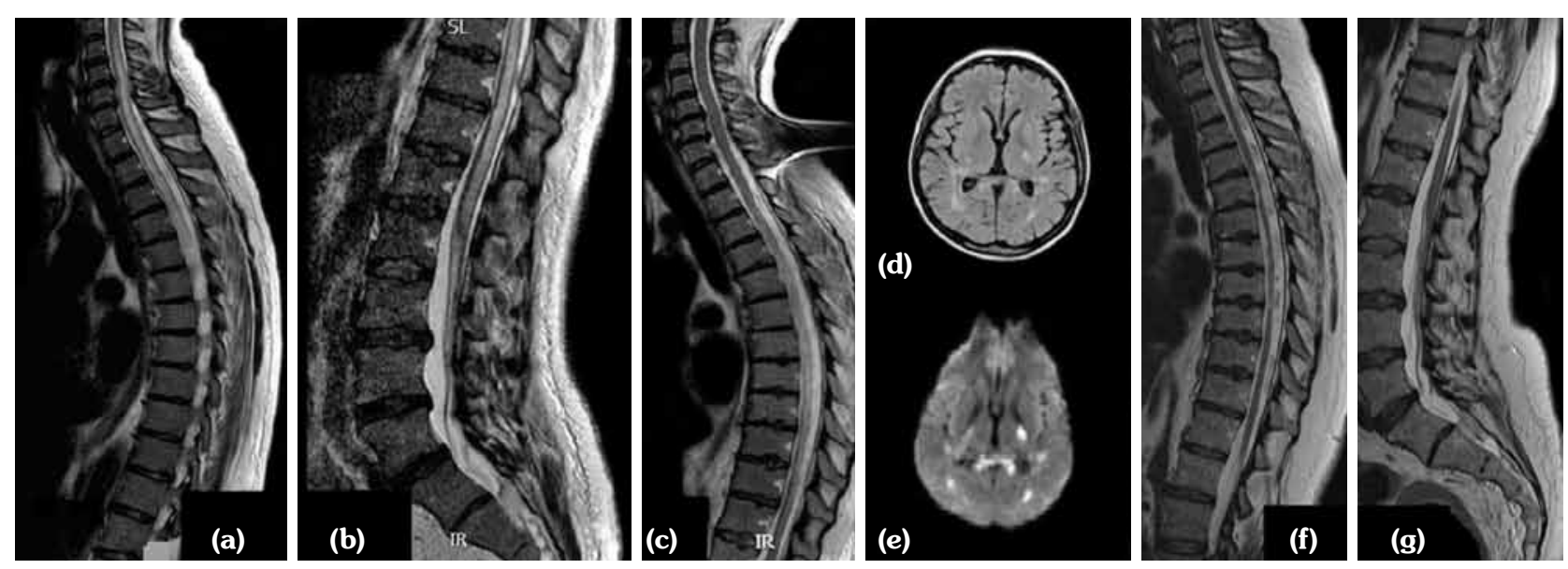

Figure 1. $T_{2}$-weighted images of the spine $(\mathbf{a}, \mathbf{b})$ before and $(\mathbf{c})$ after steroid and immunosuppressant treatment, $(\mathbf{d})$ $\mathrm{T}_{2}$-weighted and (e) diffusion-weighted imaging of the brain. Hyperintense signal visible in the (a) cervical, (b) thoracic and lumbar spine and (c) correlating with upper limbs paresis improved signal in cervical and upper thoracic spine -longitudinal myelitis. (d, e) Foci of vascular origin secondary to vasculitis in course of lupus. (f, $\mathbf{g})$ Follow-up $\mathrm{T}_{2}$-weighted images of thoracic and lumbar spine- atrophy of the spine with preservation of the cone. 
They also showed that there are no differences in the frequency of nervous system affection between SS/SLE and SLE. In a study by Kovacs et al.,11 it was stated that myelitis develops in about $2 \%$ of SLE cases, but only in $22 \%$ of the cases complete recovery is observed.

Longitudinal myelitis is defined as transverse myelitis affecting at least three adjacent segments of the spine. ${ }^{3}$ This type of myelitis is rarely observed in lupus and, according to an analysis performed by Espinosa, ${ }^{12}$ there were only 22 cases diagnosed before 2008. Still, myelitis may be the very first symptom of lupus. ${ }^{13,14}$ Interesting observations were performed by Birnbaum et al. ${ }^{13}$ where they divided myelitis into two subtypes in terms of initial symptoms, abnormalities of the grey or white matter of the spine, and the final prognosis.

In our patient, the SLE/SS overlap syndrome, manifesting with severe longitudinal myelitis, aseptic meningitis, and brain vasculitis, was diagnosed. Symptoms of myelitis correspond to affection of the grey matter of the spine and remain in good agreement with the results of Birnbaum ${ }^{13}$ as regards clinical picture and prognosis as well as represent the first signs of lupus. The patient fulfills American College of Rheumatology criteria for SLE diagnosis - malar rash, history of leucopenia and arthritis, neurological deficit, and immunological disorders ${ }^{15}$ with symptoms typical for neuropsychiatric SLE including aseptic meningitis, myelopathy, acute consciousness disturbances, and cerebral vasculitis. ${ }^{2}$ Except for clinical features and additional test results, $\mathrm{SS}$ is characterized by the presence of SSA, SSB, or antinuclear antibodies. ${ }^{16}$ An immunological marker confirming the diagnosis of the SLE/SS overlap syndrome is the presence of anti-SS related antigen B antibody. ${ }^{1}$

Given the prevalence of longitudinal myelitis in connective tissue diseases, SLE is the first to consider as a cause of this myelopathy. ${ }^{4-6,12}$ Neuromyelitis optica has been considered in differential diagnosis. Our patient does not meet the criteria proposed by Wingerchuk et al. ${ }^{17}$ since no optic nerve inflammation was detected, yet other symptoms of central nervous system affection were shown, such as meningeal syndrome and confusion, which are not typical for neuromyelitis optica. The other possible causes of myelitis ${ }^{18}$ were excluded during the diagnosis (i.e. infectious background) or due to irrelevant history (vaccinations or parasites).

\section{Declaration of conflicting interests}

The authors declared no conflicts of interest with respect to the authorship and/or publication of this article.

\section{Funding}

The authors received no financial support for the research and/or authorship of this article.

\section{REFERENCES}

1. Iaccarino L, Gatto M, Bettio S, Caso F, Rampudda $\mathrm{M}$, Zen $\mathrm{M}$, et al. Overlap connective tissue disease syndromes. Autoimmun Rev 2013;12:363-73.

2. The American College of Rheumatology nomenclature and case definitions for neuropsychiatric lupus syndromes. Arthritis Rheum 1999;42:599-608.

3. Scott TF, Frohman EM, De Seze J, Gronseth GS, Weinshenker BG. Evidence-based guideline: clinical evaluation and treatment of transverse myelitis: report of the Therapeutics and Technology Assessment Subcommittee of the American Academy of Neurology. Neurology 2011;77:2128-34.

4. Richard Mornas A, Thomas T, Pallot Prades B, Chopin F, Raoux D. Longitudinal myelitis in a patient with systemic lupus erythematosus. Joint Bone Spine 2010;77:181-3.

5. Yamamoto T, Ito S, Hattori T. Neurological picture. Acute longitudinal myelitis as the initial manifestation of Sjögren's syndrome. J Neurol Neurosurg Psychiatry 2006;77:780.

6. Katramados AM, Rabah R, Adams MD, Huq $\mathrm{AH}$, Mitsias PD. Longitudinal myelitis, aseptic meningitis, and conus medullaris infarction as presenting manifestations of pediatric systemic lupus erythematosus. Lupus 2008;17:332-6.

7. Manoussakis MN, Georgopoulou C, Zintzaras E, Spyropoulou M, Stavropoulou A, Skopouli FN, et al. Sjögren's syndrome associated with systemic lupus erythematosus: clinical and laboratory profiles and comparison with primary Sjögren's syndrome. Arthritis Rheum 2004;50:882-91.

8. Szanto A, Szodoray P, Kiss E, Kapitany A, Szegedi G, Zeher M. Clinical, serologic, and genetic profiles of patients with associated Sjögren's syndrome and systemic lupus erythematosus. Hum Immunol 2006;67:924-30.

9. Yang $Y$, Li Z, Wang L, Zhang F. The clinical and laboratory characteristics of Sjögren's syndrome that progresses to systemic lupus erythematosus: a retrospective case-control study. Int $\mathrm{J}$ Rheum Dis 2013;16:173-7. 
10. Pan HF, Ye DQ, Wang Q, Li WX, Zhang N, Li XP, et al. Clinical and laboratory profiles of systemic lupus erythematosus associated with Sjögren syndrome in China: a study of 542 patients. Clin Rheumatol 2008;27:339-43.

11. Kovacs B, Lafferty TL, Brent LH, DeHoratius RJ. Transverse myelopathy in systemic lupus erythematosus: an analysis of 14 cases and review of the literature. Ann Rheum Dis 2000;59:120-4.

12. Espinosa G, Mendizábal A, Mínguez S, Ramo-Tello C, Capellades J, Olivé A, et al. Transverse myelitis affecting more than 4 spinal segments associated with systemic lupus erythematosus: clinical, immunological, and radiological characteristics of 22 patients. Semin Arthritis Rheum 2010;39:246-56.

13. Birnbaum J, Petri M, Thompson R, Izbudak I, Kerr D. Distinct subtypes of myelitis in systemic lupus erythematosus. Arthritis Rheum 2009;60:3378-87.
14. Saison J, Costedoat-Chalumeau N, MaucortBoulch D, Iwaz J, Marignier R, Cacoub P, et al. Systemic lupus erythematosus-associated acute transverse myelitis: manifestations, treatments, outcomes, and prognostic factors in 20 patients. Lupus 2015;24:74-81.

15. Smith EL, Shmerling RH. The American College of Rheumatology criteria for the classification of systemic lupus erythematosus: strengths, weaknesses, and opportunities for improvement. Lupus 1999;8:586-95.

16. Fox RI. Sjögren's syndrome. Lancet 2005;366:321-31.

17. Wingerchuk DM, Lennon VA, Pittock SJ, Lucchinetti CF, Weinshenker BG. Revised diagnostic criteria for neuromyelitis optica. Neurology 2006;66:1485-9.

18. Bhat A, Naguwa S, Cheema G, Gershwin ME. The epidemiology of transverse myelitis. Autoimmun Rev 2010;9:395-9. 